The University of San Francisco

USF Scholarship: a digital repository @ Gleeson Library |

Geschke Center

Mathematics

College of Arts and Sciences

1999

\title{
Time Delay in the Kuramoto Model of Coupled Oscillators
}

\author{
Stephen M.K. Yeung \\ University of San Francisco, yeung@math.usfca.edu
}

S H. Strogatz

Follow this and additional works at: http://repository.usfca.edu/math

Part of the Mathematics Commons

\section{Recommended Citation}

M. K. Stephen Yeung and Steven H. Strogatz. Time Delay in the Kuramoto Model of Coupled Oscillators. Phys. Rev. Lett. 82, 648 (1999). Copyright American Physical Society.

This Article is brought to you for free and open access by the College of Arts and Sciences at USF Scholarship: a digital repository @ Gleeson Library | Geschke Center. It has been accepted for inclusion in Mathematics by an authorized administrator of USF Scholarship: a digital repository @ Gleeson Library $\mid$ Geschke Center. For more information, please contact repository@usfca.edu. 


\title{
Time Delay in the Kuramoto Model of Coupled Oscillators
}

\author{
M. K. Stephen Yeung and Steven H. Strogatz \\ Department of Theoretical and Applied Mechanics, Kimball Hall, Cornell University, Ithaca, New York 14853-1503
} (Received 13 July 1998)

\begin{abstract}
We generalize the Kuramoto model of coupled oscillators to allow time-delayed interactions. New phenomena include bistability between synchronized and incoherent states, and unsteady solutions with time-dependent order parameters. We derive exact formulas for the stability boundaries of the incoherent and synchronized states, as a function of the delay, in the special case where the oscillators are identical. The experimental implications of the model are discussed for populations of chirping crickets, where the finite speed of sound causes communication delays, and for physical systems such as coupled phase-locked loops or lasers. [S0031-9007(98)08184-8]
\end{abstract}

PACS numbers: 87.10.+e, 02.30.Ks, 05.45.+b

The Kuramoto model was originally developed as a tractable mean-field model of coupled biological oscillators [1], such as groups of chorusing crickets [2], flashing fireflies [3], and cardiac pacemaker cells [4]. In a beautiful analysis, Kuramoto showed that the model exhibits a spontaneous transition from incoherence to collective synchronization, as the coupling strength is increased past a certain threshold [5]. The model has since been analyzed more deeply and extended in various ways [6-10]. It has also been linked to several physical problems, including Landau damping in plasmas [8], the dynamics of Josephson junction arrays [11], bubbly fluids [12], and coupled Brownian ratchets [13].

Here we explore the effects of time delay on the dynamics of the Kuramoto model. In the past, delay has often been neglected in models of coupled oscillators. In many cases this approximation is physically justified, and in all cases it simplifies the mathematics. But recently several authors have begun to investigate oscillator systems where delays are not negligible $[14,15]$, motivated by neural networks where synaptic, dendritic, and propagation delays can be significant. Other authors have considered delays in systems of limit-cycle oscillators [16], with applications to arrays of lasers and microwave oscillators.

Intuitively, the problem is similar to that faced by the fans sitting in an enormous football stadium, all of whom (we suppose) are trying to clap in unison. Even if everyone were successfully clapping in perfect synchrony, it would not sound that way to the fans themselves, as the applause coming from far across the field would be significantly delayed, because of the finite speed of sound.

Nevertheless, we show that perfect synchrony is possible in the Kuramoto model with time delay, if all oscillators are identical. In fact, there can be several different synchronized states, and they can coexist with a stable incoherent state where the oscillators are completely disorganized. These multistabilities are qualitatively new: they do not occur in the original Kuramoto model.

We consider a system of phase oscillators with noisy, randomly distributed intrinsic frequencies, and with de- layed mean-field coupling:

$$
\begin{aligned}
\dot{\theta}_{i}(t)= & \omega_{i}+\xi_{i}(t) \\
& +\frac{K}{N} \sum_{j=1}^{N} \sin \left[\theta_{j}(t-\tau)-\theta_{i}(t)-\alpha\right],
\end{aligned}
$$

for $i=1, \ldots, N$. Here $\theta_{i}(t)$ is the phase of the $i$ th oscillator at time $t$, and $\omega_{i}$ is its intrinsic frequency, randomly drawn from a probability density $g(\omega)$ with mean $\omega_{0}$. The white noise $\xi_{i}(t)$ represents frequency fluctuations at an effective temperature $D \geq 0$, and is defined by the ensemble averages $\left\langle\xi_{i}(t)\right\rangle=0,\left\langle\xi_{i}(s) \xi_{j}(t)\right\rangle=2 D \delta_{i j} \delta(s-$ $t)$. In the global coupling term, $K \geq 0$ is the coupling strength, $\tau>0$ is the delay, and $\alpha$ is a phase frustration parameter. This model reduces to the Kuramoto model [5] if $\tau=0, \alpha=0$, and $D=0$, and to the mean-field $\mathrm{XY}$ model if $\tau=0, \alpha=0$, and the oscillators are identical, i.e., $g(\omega)=\delta\left(\omega-\omega_{0}\right)$. For $\tau=0$, the separate effects of frustration $\alpha$ and noise $D$ have been studied by Sakaguchi and Kuramoto [6].

As the one-parameter family of rotating-frame transformations $\theta_{i}(t) \rightarrow \theta_{i}(t)-\Omega t, \omega_{i} \rightarrow \omega_{i}-\Omega, \alpha \rightarrow \alpha+$ $\Omega \tau$ leave Eq. (1) invariant for any $\Omega$, we may assume $\alpha=0$ without loss of generality - except if $\tau=0$, which we forbid. (This restriction is merely for convenience. All of our results are well-behaved as $\tau \rightarrow 0$ and converge to those obtained by setting $\tau=0$ from the start.) Moreover, since Eq. (1) is invariant under the reflection $\omega_{i} \rightarrow-\omega_{i}$, $\theta_{i} \rightarrow-\theta_{i}, \alpha \rightarrow-\alpha$, it suffices to consider $\omega_{0} \geq 0$.

It is often helpful to describe the macroscopic state of the system in terms of the complex order parameter $R(t) e^{i \psi(t)}=\frac{1}{N} \sum_{j=1}^{N} e^{i \theta_{j}(t)}$ introduced by Kuramoto [5]. Here $R(t)$ measures the system's phase coherence. In particular, $R=1$ if all of the oscillators are in phase, whereas $R=0$ if the oscillators are scattered around the unit circle with their centroid at the origin.

Our first analytical result concerns the stability of the incoherent state for the infinite- $N$ limit of Eq. (1). We rewrite the model as a Fokker-Planck equation for the density $\rho(\theta, \omega, t)$ of oscillators currently at phase $\theta$, with 
intrinsic frequency $\omega$. Because the method is standard $[7,8]$, we omit the details. The only new feature here is that the drift velocity field inherits the time delay in Eq. (1). When the Fokker-Planck equation is linearized about the incoherent state (the stationary density $\rho \equiv$ $1 / 2 \pi)$, we find [17] that its continuous spectrum is $\{-D-$ $i \omega \mid \omega \in \operatorname{supp}(g)\}$. Hence, for $D>0$, the continuous spectrum corresponds to damped modes and therefore the stability of the incoherent state is determined solely by the discrete eigenvalues. But, when $D=0$, the continuous spectrum is pure imaginary and corresponds to neutrally stable rotating waves in the full system. In this case, the incoherent state can never be linearly stable: it is either unstable or neutral, depending on the discrete eigenvalues. These eigenvalues $\lambda$ satisfy [17]

$$
e^{-\lambda \tau} \frac{K}{2} \int_{-\infty}^{\infty} d \omega \frac{g(\omega)}{\lambda+D+i \omega}=1 .
$$

This implicit formula for $\lambda$ is exact but difficult to analyze for arbitrary $g(\omega)$, so we consider the case of identical oscillators to gain some insight. Even this case turns out to be far from trivial. If $g(\omega)=\delta\left(\omega-\omega_{0}\right)$, Eq. (2) can be simplified to the transcendental equation

$$
(p+i r-z) e^{z}+q=0,
$$

where $p=-D \tau \leq 0, r=-\omega_{0} \tau, q=K \tau / 2$, and $z=$ $\lambda \tau$. Then the stability of the incoherent state depends on whether all roots of Eq. (3) satisfy $\operatorname{Re}(z)<0$ (in which case we will say "all eigenvalues are stable," for brevity).

By the transformations $z \rightarrow z+i n \pi, q \rightarrow(-1)^{n} q$, $r \rightarrow r+n \pi$, and $z \rightarrow z^{*}, r \rightarrow-r$, we may assume $r \in$ $[0, \pi / 2]$ in Eq. (3). For $r=0$, Hayes proved $[18,19]$ that all eigenvalues are stable if and only if $p<1$ and $p<-q<\sqrt{p^{2}+y_{1}^{2}}$, where $y_{1}$ is the unique zero of $p \sin y-y \cos y$ in $(0, \pi)$. Using results of Pontryagin as in Ref. [19], we can show [17,20] the following for $r \in(0, \pi / 2]$.

(i) If $p=0$, then all eigenvalues are stable if and only if $r-\pi / 2<q<0$.

(ii) If $p<0$, then all eigenvalues are stable if and only if $-\sqrt{p^{2}+\left(y_{2}-r\right)^{2}}<q<\sqrt{p^{2}+\left(y_{1}-r\right)^{2}}$, where $y_{1}$ and $y_{2}$ are the unique zeros of $p \sin y+(r-y) \cos y$ in $(0, r)$ and $(\pi / 2, \pi)$, respectively.

These conditions are exact but still opaque, so we simplify the model further for illustration. Suppose there is no noise $(D=0)$. Then we find [17] that the incoherent state is neutrally stable precisely when

$$
K<\frac{\omega_{0}}{2 m-1} \text { and } \frac{(4 m-3) \pi}{2 \omega_{0}-K}<\tau<\frac{(4 m-1) \pi}{2 \omega_{0}+K},
$$

with $m$ being an arbitrary positive integer.

Figure 1 shows that this analytical result agrees with numerical simulations, even for as few as $N=12$ oscillators. Although the incoherent state is neutrally stable in the grey region, we observe numerically that $R(t) \rightarrow 0 \mathrm{ex}-$

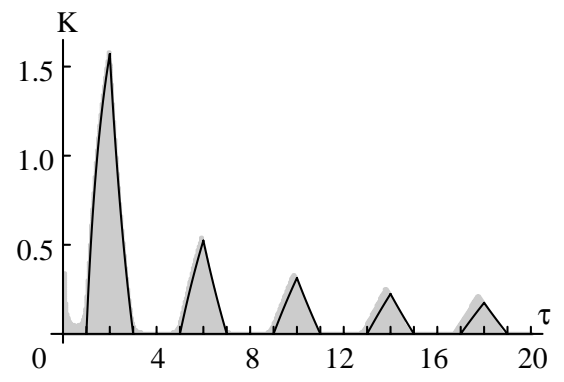

FIG. 1. Stability region for the incoherent state, with $g(\omega)=$ $\delta\left(\omega-\omega_{0}\right), \quad \omega_{0}=\pi / 2, \quad D=0, \quad N=12$. Black curves: theoretical boundaries (4) for the infinite- $N$ limit; grey area: results from numerical integration using a sixth order AdamsBashforth-Moulton scheme, with fixed stepsize $d t=\tau / 20$, and with the corrector formula iterated for convergence and stability. Initially, all of the phases were evenly spaced, and the symmetry was broken by adding $O\left(10^{-10}\right)$ random perturbations. The incoherent state was judged as unstable if $R(t)>10^{-7}$ at a final time of $t=800 \tau$.

ponentially fast, as in Landau damping [8]. In this sense, incoherence is stable in the grey region.

Continuing with the instructive case of noiseless, identical oscillators, we now consider the possibility of perfect synchrony: $\theta_{i}(t)=\theta(t)$ for all $i$. We restrict our attention to a particular class of such solutions, namely, uniform rotations: $\theta(t)=\Omega t+\beta$. Self-consistency then requires

$$
\Omega=\omega_{0}-K \sin (\Omega \tau),
$$

for such solutions to exist, and linearization [17] imposes

$$
\cos (\Omega \tau)>0,
$$

as the condition for their orbital stability.

If we graph both sides of Eq. (5) as functions of $\Omega$, we see that for all sufficiently large $K$, there exist multiple stable synchronized states [21], as Eq. (5) has nonunique solutions satisfying (6). We can also see that stable synchrony is impossible for certain combinations of $\tau$ and $K$. The problem reduces to characterizing the twoparameter family of lines of negative slope that intersect the sine function on its descending limbs. We find that stable synchronized states do not exist if and only if

$$
\begin{aligned}
K & <\frac{\omega_{0}}{2(2 m-1)} \text { and } \\
\frac{(4 m-3) \pi}{2 \omega_{0}-2 K} & <\tau<\frac{(4 m-1) \pi}{2 \omega_{0}+2 K},
\end{aligned}
$$

with $m$ being an arbitrary positive integer.

These zones of forbidden synchrony are shown in black in Fig. 2. For comparison, they are overlaid on top of the earlier grey regions (Fig. 1) where incoherence is stable. The black regions fit neatly inside the grey; they have the same base and half the height. The exposed parts of the grey regions correspond to bistability: stable incoherence coexists with stable synchrony, and hysteresis can occur. 


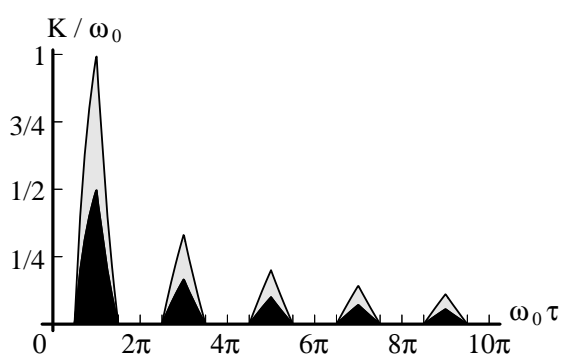

FIG. 2. Stability regions of the incoherent state (4) and the synchronized states (7) for $g(\omega)=\delta\left(\omega-\omega_{0}\right), D=0$. White region: one or more stable synchronized states exist but the incoherent state is unstable; black region: incoherence is stable but synchrony is not; grey region: one or more stable synchronized states coexist with stable incoherence.

Numerical simulations reveal windows in the bistable regions where $R$ can be time periodic (Fig. 3). In the example shown, period doubling occurs as $K$ increases, but seems to be truncated beyond period 16 (not shown); after that, a synchronized state apparently takes over, suppressing further period doubling [Fig. 3(d)]. Such unsteady behavior is a consequence of the delay; in the standard Kuramoto model, numerical experiments show that $R(t)$ always approaches a constant value if $g(\omega)$ is unimodal and symmetric (although this has never been proven). Oscillator configurations with two or more clusters [22] cause the unsteady behavior seen here. All of the clusters move with the same average velocity, but their separation is periodically modulated.

So far we have concentrated on identical oscillators. To check how the results would be modified for other frequency distributions, we have considered the Lorentzian distribution $g(\omega)=(\gamma / \pi)\left[\gamma^{2}+\left(\omega-\omega_{0}\right)^{2}\right]^{-1}$. Then by a remarkable coincidence [17], Eq. (2) can again be reduced to Eq. (3), but now with $p=-(\gamma+D) \tau, r=$ $-\omega_{0} \tau, q=K \tau / 2$, and $z=\lambda \tau$. The critical coupling is given by

$$
K_{c}=2(\gamma+D) \sec \left(\Omega_{c} \tau\right),
$$
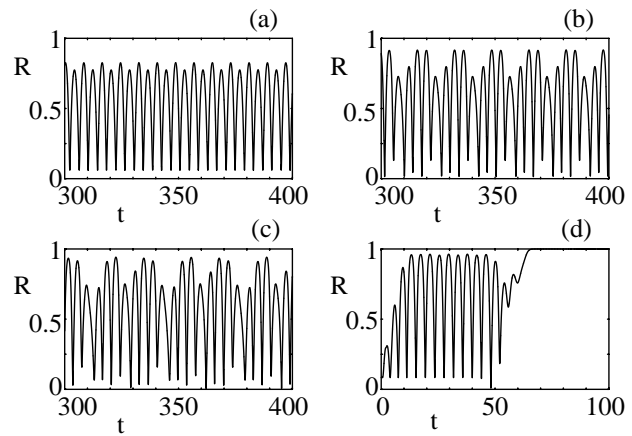

FIG. 3. Time series showing nonsteady order parameter $R(t)$, with $g(\omega)=\delta\left(\omega-\omega_{0}\right), \omega_{0}=\pi / 2, D=0, \tau=2, N=$ 24. (a) $K=1.3$ : period-2 oscillation; (b) $K=1.4$ : period-4 oscillation; (c) $K=1.44$ : period-8 oscillation; (d) $K=1.475$ : $R(t) \rightarrow 1$ after a periodic transient. where $\Omega_{c}=\omega_{0}-(\gamma+D) \tan \left(\Omega_{c} \tau\right)$. Figure 4 plots the corresponding region where incoherence is stable. It resembles Fig. 1, with a series of evenly spaced peaks [at $\omega_{0} \tau=(2 n+1) \pi$ ] that decrease in height. The main difference is that the distributed frequencies produce some rounding of the boundary, and lift it off the $\tau$ axis so that it now has minima $2(\gamma+D)$ at $\omega_{0} \tau=2 n \pi$.

The bistability found earlier also has a counterpart in the Lorentzian case [Fig. 4(a)]. Partially locked states (which may not be unique) replace the earlier in-phase states, but otherwise the story is unchanged [23]. Thus, the case of identical oscillators captures the essential features introduced by delay.

Our final result concerns the bifurcation at $K=K_{c}$, where the incoherent state becomes unstable. We have adapted the two-timing method of Ref. [9] to handle the delay-differential equations (1). We find [17] that generically, for $D \geq 0$ and arbitrary $g(\omega)$, a Hopf bifurcation occurs at $K_{c}$ [24], giving rise to a partially locked state, or in the density description, a rotating wave with constant coherence $R=O\left(\sqrt{\left|K-K_{c}\right|}\right)$. This bifurcation may be subcritical [Fig. 4(a)] or supercritical [Fig. 4(b)].

Experimental tests of the model may be possible in networks of phase-locked loops [25], relativistic magnetrons [26], solid-state lasers [27], or communication satellites [28], as they are all approximately governed by coupled Adler equations [29] similar in form to Eq. (1). The delay and the coupling strength are both natural control parameters, and perhaps one could try to map out the stability boundaries, look for hysteresis between incoherence and synchrony, etc. Our model may also help to explain how crickets can synchronize their chirps [2], despite the time delays caused by the speed of sound. Crickets listen to each other's chirps and adjust their own timing according to a phase response curve [2]. The propagation delay between two crickets $3 \mathrm{~m}$ apart is about $10 \mathrm{msec}$. This is

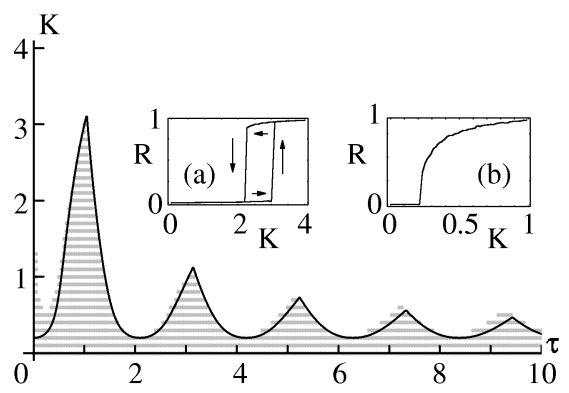

FIG. 4. Stability region of the incoherent state for Lorentzian $g(\omega)$ with $\omega_{0}=3, \gamma=0.1, D=0, N=3600$. Black curves: theoretical boundary Eq. (8); grey stripes: numerical results using the same method as in Fig. 1. Insets: (a) Subcritical Hopf bifurcation of the incoherent state at $\tau=1$. Arrows indicate a hysteresis loop between stable incoherence and a stable partially locked state with $R$ close to 1 . (b) Supercritical Hopf bifurcation of the incoherent state at $\tau=2$. A stable partially locked state grows continuously from the incoherent state with $R=O\left(\sqrt{K-K_{c}}\right)$. 
short compared to the chirp period (300-500 msec). Our results suggest that delay effects become significant only in the first peak in Fig. 2, i.e., for delays near half the period of the oscillation. Thus the delays that crickets actually encounter in the field are probably negligible as far as synchrony is concerned. It would be interesting to try lab experiments on crickets interacting via chirp signals whose delay and amplitude can be electronically manipulated.

This research was supported in part by the National Science Foundation. We thank Tim Forrest for information about crickets.

[1] A. T. Winfree, J. Theor. Biol. 16, 15 (1967).

[2] T. J. Walker, Science 166, 891 (1969); E. Sismondo, ibid. 249, 55 (1990).

[3] J. Buck, Q. Rev. Biol. 63, 265 (1988).

[4] C.S. Peskin, Mathematical Aspects of Heart Physiology (Courant Institute of Mathematical Sciences, New York, 1975).

[5] Y. Kuramoto, in International Symposium on Mathematical Problems in Theoretical Physics, Lecture Notes in Physics Vol. 39, edited by H. Araki (Springer-Verlag, Berlin, 1975); Chemical Oscillations, Waves, and Turbulence (Springer-Verlag, Berlin, 1984).

[6] H. Sakaguchi and Y. Kuramoto, Prog. Theor. Phys. 76, 576 (1986); H. Sakaguchi, ibid. 79, 39 (1988).

[7] S. H. Strogatz and R.E. Mirollo, J. Stat. Phys. 63, 613 (1991); J.D. Crawford, J. Stat. Phys. 74, 1047 (1994); Phys. Rev. Lett. 74, 4341 (1995).

[8] S. H. Strogatz, R.E. Mirollo, and P.C. Matthews, Phys. Rev. Lett. 68, 2730 (1992).

[9] L. L. Bonilla, C. J. Pérez Vicente, and R. Spigler, Physica (Amsterdam) 113D, 79 (1998).

[10] B. Ermentrout, J. Math. Biol. 22, 1 (1985); H. Daido, Phys. Rev. Lett. 73, 760 (1994); Physica (Amsterdam) 91D, 24 (1996); H.-A. Tanaka, A. J. Lichtenberg, and S. Oishi, Phys. Rev. Lett. 78, 2104 (1997); J. A. Acebrón, L. L. Bonilla, S. D. Leo, and R. Spigler, Phys. Rev. E 57, 5287 (1998).

[11] K. Wiesenfeld, P. Colet, and S. H. Strogatz, Phys. Rev. Lett. 76, 404 (1996); Phys. Rev. E 57, 1563 (1998).

[12] G. Russo and P. Smereka, SIAM J. Appl. Math. 56, 327 (1996); P. Smereka (to be published).

[13] R. Häußler, R. Bartussek, and P. Hänggi, in Applied Nonlinear Dynamics and Stochastic Systems near the Millenium, edited by J.B. Kadtke and A. Bulsara, AIP Conf. Proc. No. 411 (AIP, New York, 1997), pp. 243248.

[14] E. Niebur, H. G. Schuster, and D. M. Kammen, Phys. Rev. Lett. 67, 2753 (1991).
[15] H. G. Schuster and P. Wagner, Prog. Theor. Phys. 81, 939 (1989); B. Dorizzi and B. Grammaticos, Phys. Rev. A 44, 6958 (1991); W. Gerstner and J. L. van Hemmen, Phys. Rev. Lett. 71, 312 (1993); P. C. Bressloff, S. Coombes, and B. de Souza, ibid. 79, 2791 (1997); P. C. Bressloff and S. Coombes, ibid. 80, 4815 (1998); S. Kim, S. H. Park, and C. S. Ryu, ibid. 79, 2911 (1997); Y. Nakamura, F. Tominaga, and T. Munakata, Phys. Rev. E 49, 4849 (1994); C. van Vreeswijk, ibid. 54, 5522 (1996); U. Ernst, K. Pawelzik, and T. Geisel, ibid. 57, 2150 (1998); T. B. Luzyanina, Network-Comp. Neural 6, 43 (1995); A. Nischwitz and H. Glünder, Biol. Cybernet. 73, 389 (1995); E. M. Izhikevich, Phys. Rev. E 58, 905 (1998).

[16] J. J. Lynch and R. A. York, IEEE Trans. Circuits Syst. I 42, 413 (1995); S. Wirkus and R. Rand, in ASME Design Engineering Technical Conferences (American Society of Mechanical Engineers, New York, 1997); D. V. R. Reddy, A. Sen, and G. L. Johnston, Phys. Rev. Lett. 80, 5109 (1998).

[17] M. K. S. Yeung and S. H. Strogatz (to be published).

[18] N. D. Hayes, J. London Math. Soc. 25, 226 (1950).

[19] R. Bellman and K. L. Cooke, Differential-Difference Equations (Academic Press, New York, 1963).

[20] Physically, $p=-D \tau \leq 0$. But see Ref. [17] for the case $p>0$, as well as generalizations of Eq. (3).

[21] In Ref. [14] Niebur et al. analyzed the case of large $K$ and small $\tau$ for a two-dimensional network with nearestneighbor coupling. They found an equation analogous to Eq. (5) and that the smallest root corresponded to the most stable solution. This is consistent with (6). However, if $\omega_{0} \tau$ is not small, the smallest root of Eq. (5) may not be a stable frequency [as dictated by (6)].

[22] D. Golomb, D. Hansel, B. Shraiman, and H. Sompolinsky, Phys. Rev. A 45, 3516 (1992); K. Okuda, Physica (Amsterdam) 63D, 424 (1993); K. Kaneko, ibid. 75D, 55 (1994).

[23] However, we have not seen unsteady $R(t)$ in simulations for Lorentzian $g(\omega)$.

[24] At special parameter values, e.g., the turning points $\omega_{0} \tau=n \pi$ of the stability boundary in Fig. 4, Eq. (1) is $O(2)$ symmetric and codimension-2 bifurcations occur.

[25] Phase-Locked Loops and Their Applications, edited by W. C. Lindsey and M. K. Simon (IEEE Press, New York, 1978).

[26] J. Benford, H. Sze, W. Woo, R. R. Smith, and B. Harteneck, Phys. Rev. Lett. 62, 969 (1989).

[27] L. Fabiny, P. Colet, R. Roy, and D. Lenstra, Phys. Rev. A 47, 4287 (1993).

[28] W. C. Lindsey and J.-H. Chen, Euro. Trans. Telecommun. 7, 25 (1996).

[29] R. Adler, Proc. IRE 34, 351 (1946); reprinted in Proc. IEEE 61, 1380 (1973). 